\title{
Characterization of Metallic Formation on Ion Exchange/Chelating Resins by Various Metal Groups and Extrapolation to Molecular Models
}

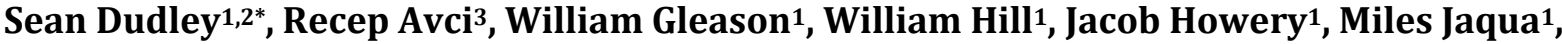 Daisy Margrave ${ }^{1}$}

${ }^{1}$ Montana Tech of the University of Montana, Butte, USA

${ }^{2}$ Jensen Hughes, Mountlake Terrace, USA

${ }^{3}$ Montana State University, Bozeman, USA

Email: *sdudley@mtech.edu

How to cite this paper: Dudley, S., Avci, R., Gleason, W., Hill, W., Howery, J., Jaqua, M. and Margrave, D. (2018) Characterization of Metallic Formation on Ion Exchange/Chelating Resins by Various Metal Groups and Extrapolation to Molecular Models. Journal of Minerals and Materials Characterization and Engineering, 6, 72-85. https://doi.org/10.4236/jmmce.2018.61007

Received: December 9, 2017

Accepted: January 26, 2018

Published: January 29, 2018

Copyright $\odot 2018$ by authors and Scientific Research Publishing Inc. This work is licensed under the Creative Commons Attribution International License (CC BY 4.0).

http://creativecommons.org/licenses/by/4.0/

\begin{abstract}
This paper examines the metallic rare earth element (REE) formations that grow on ion exchange/chelating resins. Formation of these stabilized metallic structures leads to composite particle destruction and appears to be the result of the dynamic environment of the batch experimentation. Polymeric structure, electron availability, $\mathrm{pH}$, kinetic factors, and the REE f-orbitals play significant roles in the formation of the organometallic framework. f-orbitals are largely still not understood to a great extent but this work serves to elucidate the larger role they may play in ligand interactions. Molecular modeling was utilized as a secondary component in investigating rare earth element (REE) deposition onto ion exchange/chelating resins. Modeling of the f-orbital frontier regions and the application of the HOMO-LUMO transition's effect on molecular transfer and stability is discussed. Advanced metallic loading, in the manner of an organometallic structure, shows short-term stability resulting in particle destruction as increased REE is adsorbed.
\end{abstract}

\section{Keywords}

Rare Earth, Recovery, Polymer, Modeling, Characterization

\section{Introduction}

Researchers at Montana Tech of the University of Montana are looking for costeffective ways to recover and concentrate rare earth elements (REE). Ion ex- 
change/chelating resins have been widely used by the mining and wastewater industries for selective recovery and concentration of metals. Research into Rare Earth Element (REE) recovery is of increasing concern to the United States. A major interested party is the United States Government, that utilises REE for defense applications.

There has been a push, in the United States (US), to determine dependence on rare earth metals. Rare earth metals are incorporated and used for a number of applications including magnetic materials, battery components, alloying agents, communications, transportation, and defense. From 1960 through the 1980's, the United States was the predominant producer of rare earth metals. As of a study done in 2011, China accounts for 97\% of global production; and the US has one domestic processing company and no mining operations. [1]. As of 2015 the processing company, Molycorp, declared bankruptcy.

The process of REE absorption was found to follow: 1) initial ionic attraction bonds rare earth elements to the surface of the composite material, 2) subsequent covalent interaction serves to transfer species through bridging with neighboring ligand fields to the interior of the particle. HOMO and LUMO [2] theory can be integrated to explain the transfer mechanism. The organometallic network formed delocalises electrons but does result in concentrations. Resulting bridging of ligand fields provides a substrate for metallic bonding to form. Parallel to the bridging formations a failure of inner electron transfer networks may activate an attempt to establish a new electron network through metallic formation and deposition. Eventually metallic conformations and inner organometallic electron transfer network failure induce stress and result in breakage of the particle but metallic components are capable of staying intact, but are now limited in bonding due to loss of organometallic properties.

Montana Tech is seeking to expand this technique to REE processing by testing several silica and polystyrene composites having multiple functional groups. Initial investigation showed atypical behavior in resins when loaded with REE instead of transitional metals. While metal REE structures formed on the surface of the ion exchange resins, particle destruction followed in specific cases. Work to characterize the root-causes and mechanisms of metallic deposition and breakage continues.

Previous work at Montana Tech has shown REE self-assembling on both ion exchange and chelating resins. The intent of the experimental phase reported here was to broaden both the spectrum of resins being used and the REE being loaded to determine whether this phenomenon was occurring across the spectrum of resins and REE, and to determine the form the REE take on the resins and compare that with other types of metals. Molecular modeling was used to possibly provide information explaining the phenomena being seen.

\section{Experimental}

Batch experimentation in this phase of work provided a comprehensive data set 
for characterization. Batch experiments were done on a table-top shaker set at $200 \mathrm{rpm}$. Solutions adjusted to $\mathrm{pH} 2,5,8$, and 10 were measured into scintillation vials at $20 \mathrm{ml}$. The REE chlorides were added to solutions to produce concentrations of $1000 \mathrm{ppm}$ REE. The batches were shaken and sufficient contact time allowed for full loading to occur. The material was sampled at three different stages: 24 hours, 48 hours, and after several days.

Previous research has shown that $\mathrm{pH}$ and contact time are the primary means of controlling the adsorption process. Key results of the studies, which in some cases are contradictory, are:

- Chromium(VI) onto silica-Adsorption qualities are highly dependent on $\mathrm{pH}$ conditions [3]. Most adsorption occurs within 20 minutes [4].

- Praseodymium onto pyridine/phosphate-Adsorption rate is governed by interdiffusional kinetics-rates at which elements can travel and find adsorption sites [5].

- Cerium(III) onto D151-Higher pH conditions are optimal for, suggesting that adsorption is ion- and ligand-specific [6].

- Ytterbium(III) onto weak acid gel-Low and high $\mathrm{pH}$ conditions inhibit recovery, while optimum recovery was seen at $\mathrm{pH} 5.5$ [7].

- The adsorption process is dependent on acid $\left(\mathrm{HNO}_{3}\right)$ concentration and temperature, indicating that an increase in temperature was not beneficial to adsorption.

On the basis of those studies, we chose solution conditions of $\mathrm{pH} 2$ and $\mathrm{pH} 10$ as boundaries. Because composites become unstable at extremely low or high $\mathrm{pH}$, we used $\mathrm{pH} 5$ and $\mathrm{pH} 8$ as midpoints. We found that $\mathrm{pH}$ after contact time settled around 2, and that adsorption was sufficient. This was consistent with the work of Yao, which found that as $\mathrm{pH}$ increased recovery decreased [5].

The REE we studied were chosen because of their availability and to compare REE adsorption between light and heavy elements. REE chlorides were chosen because chlorine would likely remain a bystander in chemical reactions. Temperature was not considered an important factor in this stage of experimentation.

Primary analysis for this phase of work consisted of scanning electron microscopy (SEM) with energy dispersive $\mathrm{x}$-ray spectroscopy (EDS) and $\mathrm{x}$-ray photoelectron spectroscopy (XPS).

\section{Results \& Discussion}

\subsection{Characterization of Metallic Formation}

A number of depositional morphologies were seen on both silica- and polystyrene-based ion exchange/chelating resins, depending on resin, REE, and solution $\mathrm{pH}$. The effect of the metal type on depositional morphology is addressed here with only a small portion of the work presented. Transitional metals, precious metals, and REE metals were compared. Note that these are general comparisons where characterization between SEM EDS and XPS may not be from the same 
particles.

Three common types of morphology seen with REE deposition are presented. Figure 1 shows dysprosium on polystyrene/aminophosphonic ion exchange resin. Present are the circular deposits indicative of close contact between resin particles, flake deposits only loosely bound to the surface, and connected deposits where nonrandom deposition is evident.

Figure 2 shows SEM EDS analysis of the flake structure (crosshair shown in Figure 1) and represents a common pattern for REE metal deposition onto the polystyrene/aminophosphonic ion exchange resin. In this case, all the metals identified are included even if they cannot be present. This is to illustrate the difficulties of surface analysis on round particles where linear depositions have further complicated the matter. The counts are low for standard EDS scans, but given the nature of the material, the peaks were compared. Polymer substructure and functionalization peaks are present and may, in fact, be misidentified as other minor peaks such as lutetium, gold, and uranium due to difficulties analyzing curved surfaces. Dysprosium is present in significant amounts as indicated by the peaks. Both carbon and oxygen are also identified in this case, indicating to some extent the thickness of the metal layers and how complete the coverage is. The oxygen present is not associated with the REE, as further analysis showed, but is associated with the structure of the polymer. The presence of oxygen peaks in the SEM/EDS data, but the confirmation that the REE are not oxidised highlights the complex nature of the ORP system in which the polymer was tested. Further testing may control the atmosphere utilizing an inert condition and controlling the dissolved oxygen in solution.

Figure 3 is a SEM of holmium on polystyrene/aminophosphonic ion exchange resin. Flake structures are also present in this image, as is the edge of a large circular deposition in the bottom left particle.

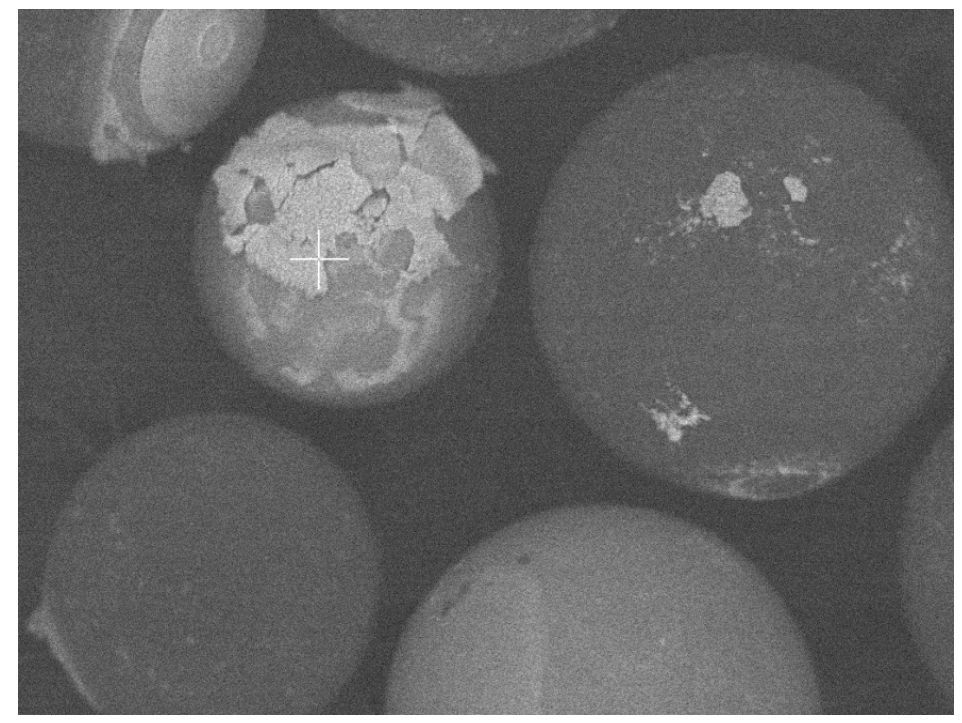

Figure 1. SEM of dysprosium on polystyrene/aminophosphonic ion exchange resin at $\mathrm{pH}$ 10. 
Label A:

Counts

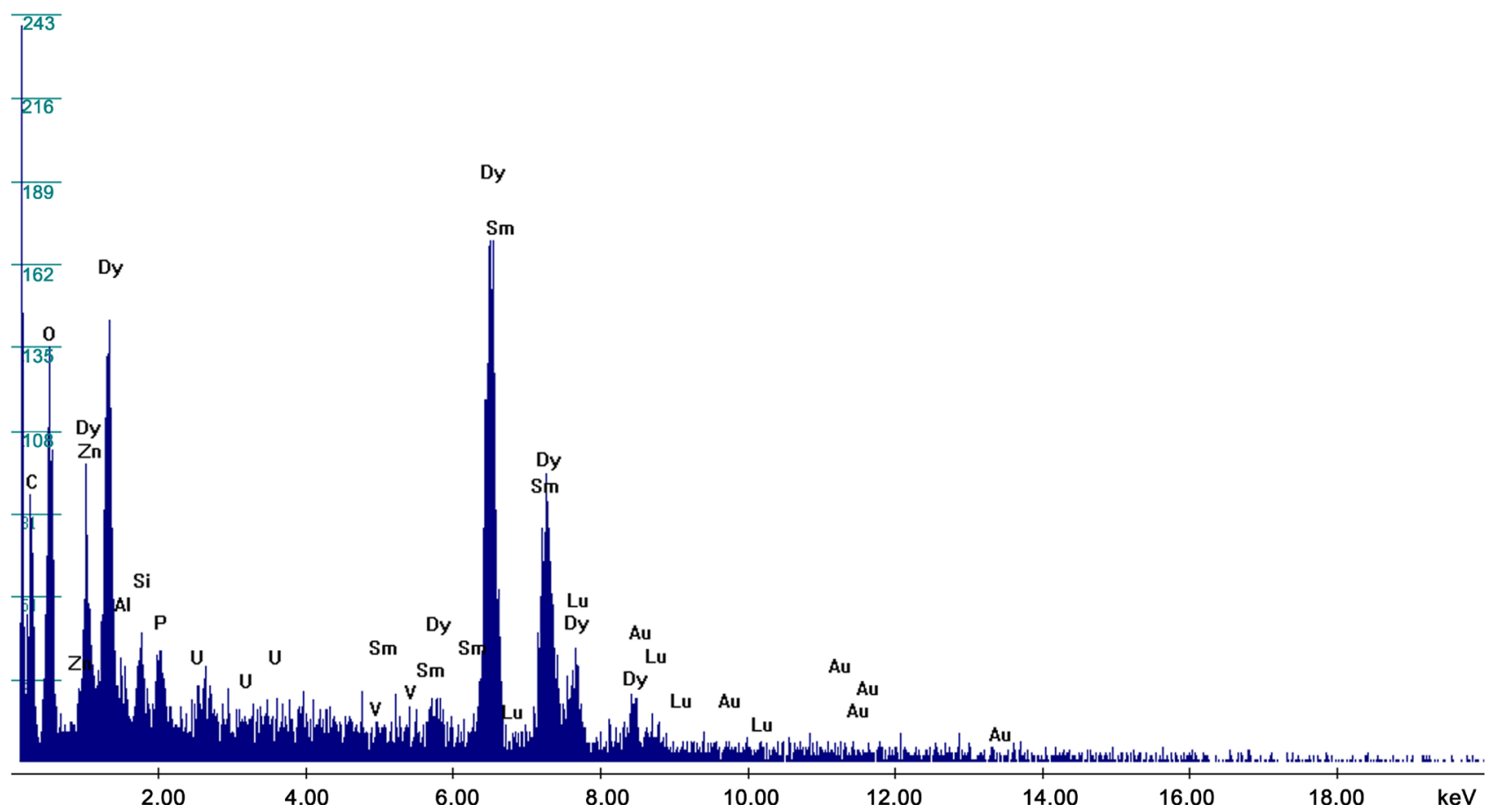

Figure 2. EDS of dysprosium on polystyrene/aminophosphonic ion exchange resin at pH 10.

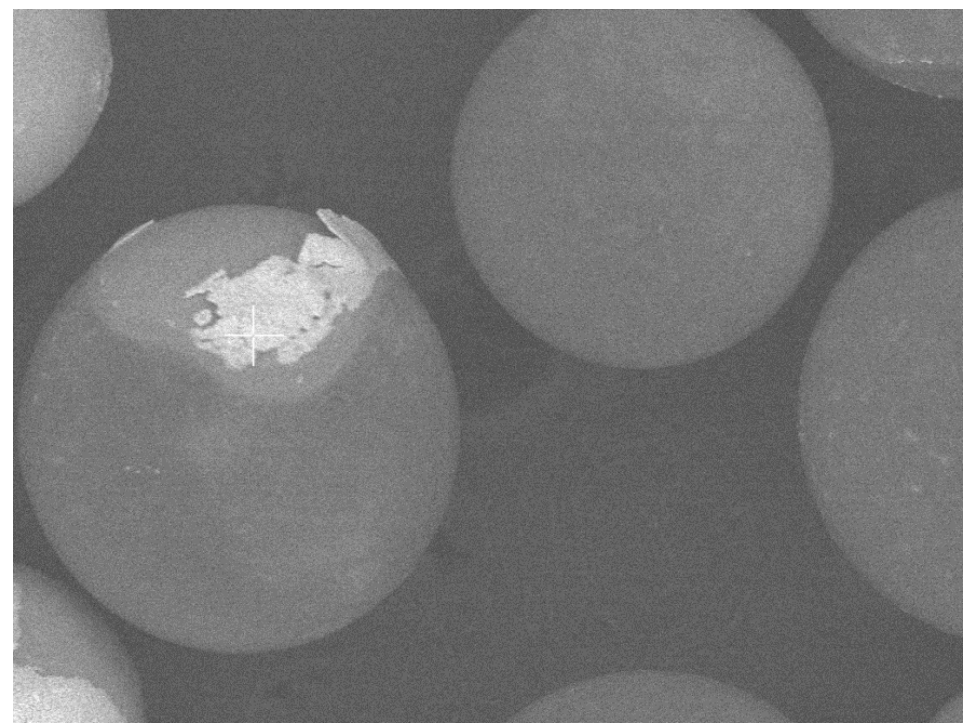

Figure 3. SEM of holmium on polystyrene/aminophosphonic ion exchange resin at $\mathrm{pH}$ 10.

Figure 4 shows the EDS analysis of the holmium deposit (crosshair in Figure 3). Another complete identification is shown. In this case, sodium and oxygen 
Label A:

Counts

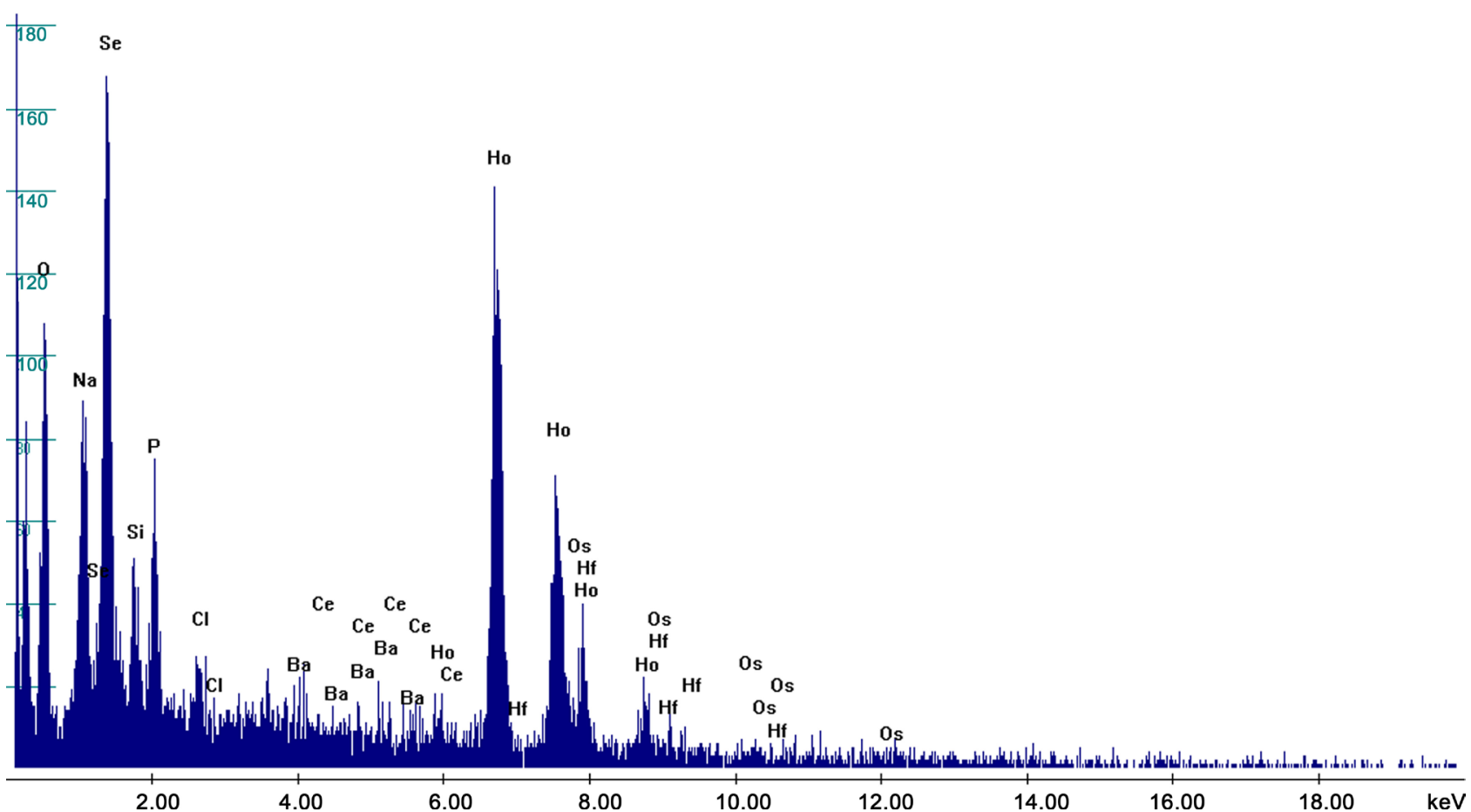

Figure 4. EDS of holmium on polystyrene/aminophosphonic ion exchange resin at $\mathrm{pH} 10$.

are identified. It should be noted that the particles are cleaned and dried before analysis so that remnants of several steps may be present. The counts are low for standard EDS scans, but given the nature of the material, the peaks were compared. Peaks of the polymer substructure and functionalization are present, again with possible interference due to difficulties analyzing curved surfaces. Of importance is the predominant holmium peak at increased counts.

Since EDS analysis showed other elements, such as oxygen, carbon, and chlorides, that may indicate nonmetallic depositions, XPS was done to confirm whether the formations within and on the surface of the ion exchange composites were metallic. Several resins were analyzed, including the polystyrene/ aminophosphonic resins. The surfaces of the particles were sputter cleaned to a depth of no more than $2 \mathrm{~nm}$ to remove any contamination due to handling.

Figure 5 and Figure 6 show the XPS spectra. The positions of the dysprosium and holmium $4 \mathrm{~d}$ and $3 \mathrm{~d} 5 / 2$ peak positions are principally where the neutral elemental peak positions of these elements would be expected. Otherwise, these peak positions would have shown large chemical shifts if these elements were to be oxidized. For example, +3 oxidation state of Dy4d peak position would be located at $168 \mathrm{eV}$ instead of $153 \mathrm{eV}$ and Dy3d5/2 would be at $\sim 1289 \mathrm{eV}$ instead of $\sim 1296 \mathrm{eV}$. Holmium is expected to show similar chemical shifts. Our measurements suggest that both dysprosium and holmium are not oxidized. The indium 


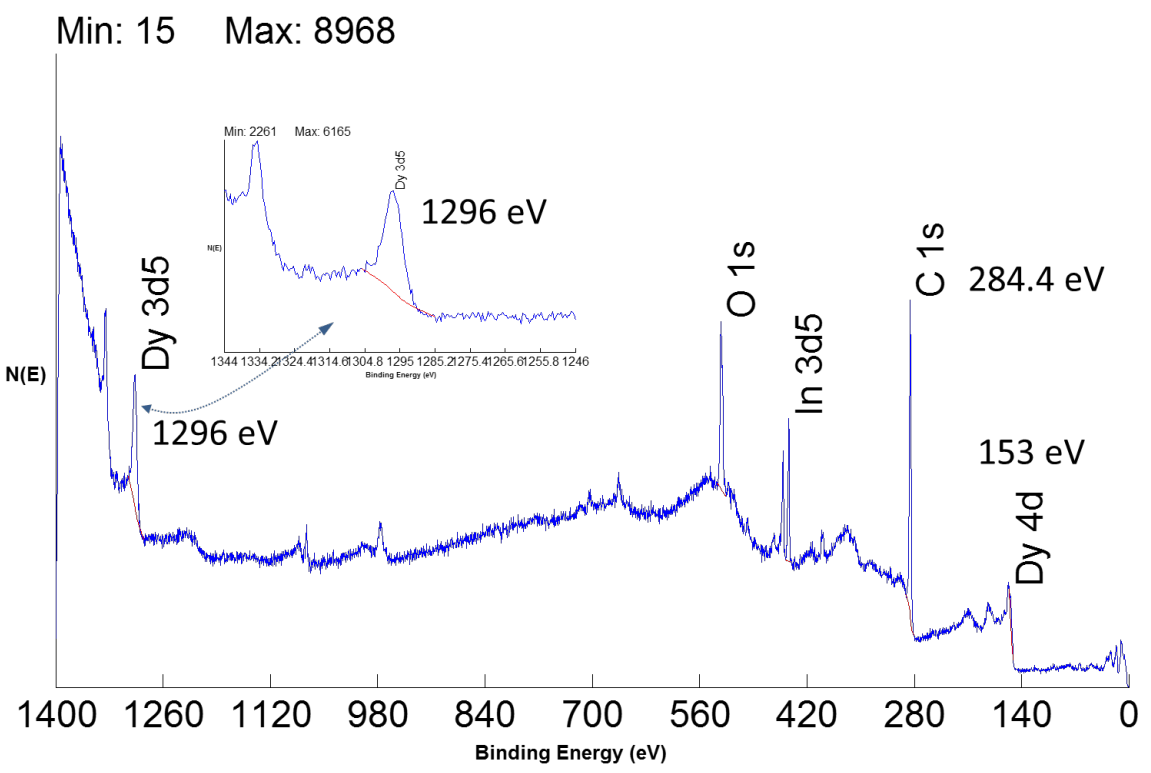

Figure 5. XPS spectrum for dysprosium on polystyrene resin at pH 5.

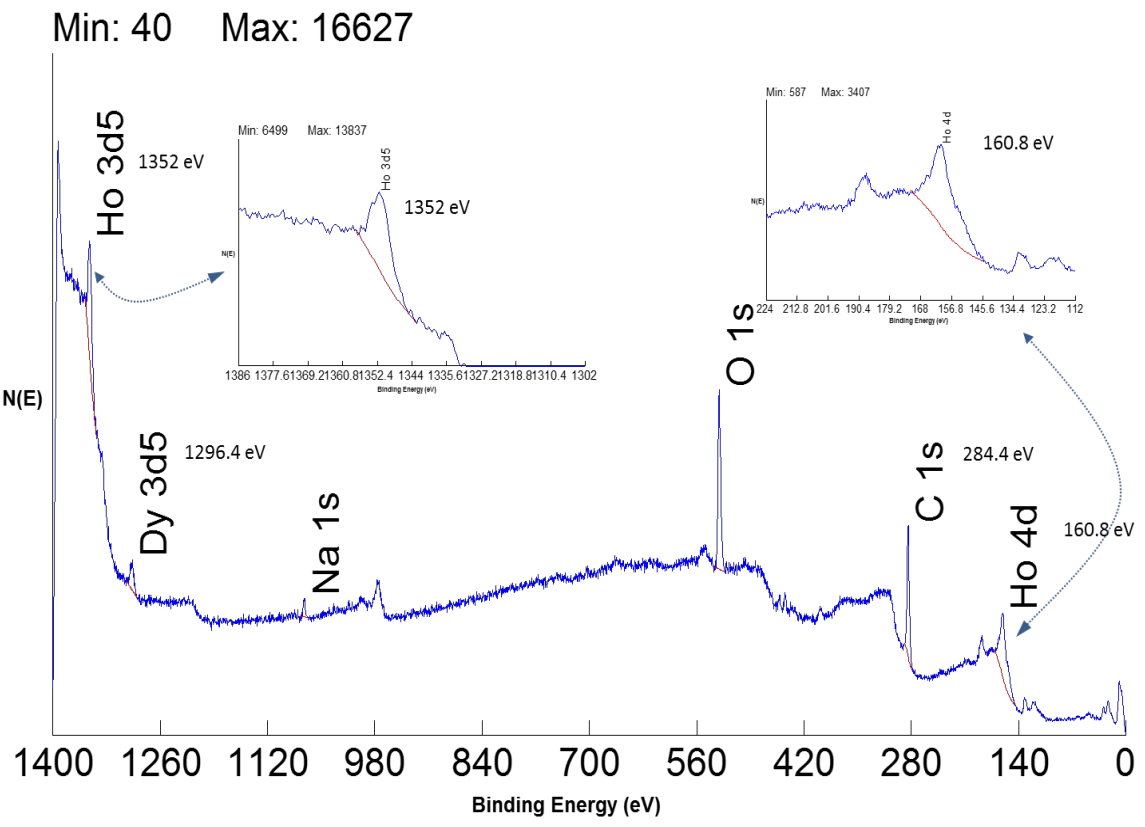

Figure 6. XPS spectrum for holmium on polystyrene resin at $\mathrm{pH} 5$.

peak is correlated to the foil used for mounting. The oxygen and carbon peaks are associated with the underlying polystyrene structure. The two dysprosium peaks identify the formation as metallic because no shift occurs. The shift would be associated with the presence of oxymetallic compounds.

Figure 6 shows the XPS spectrum for holmium on polystyrene/aminophosphonic resin. Although no holmium XPS plot is readily available for holmium oxide, it is assumed that it would act much the same as dysprosium. The oxygen, carbon, and sodium peaks are associated with the underlying polystyrene structure. The presence of dysprosium is a result of accidental resin mixing. The two holmium 
peaks show no shift from metallic holmium peaks and are therefore assumed to be metallic in nature. The XPS spectra confirm the evidence from calorimetry, XRD, and SEM EDS that metal forms.

To further test that REE deposition is unlike that of other metallic groups, we tested both transitional and precious metal depositional states starting from the chloride form and under the same experimental protocols. SEM and SEM EDS analyses of the palladium experiment are presented-results from all the nonREE metal types tested showed similar results. Figure 7 shows the deposition onto a polystyrene/aminophosphonic resin sampled at 24 hours. Note the circular formations, commonly seen on REE metallic depositions.

Figure 8 shows the deposition onto a polystyrene resin sampled at 48 hours. The deposition has assumed a random pattern with most of the surface involved in the deposition event.

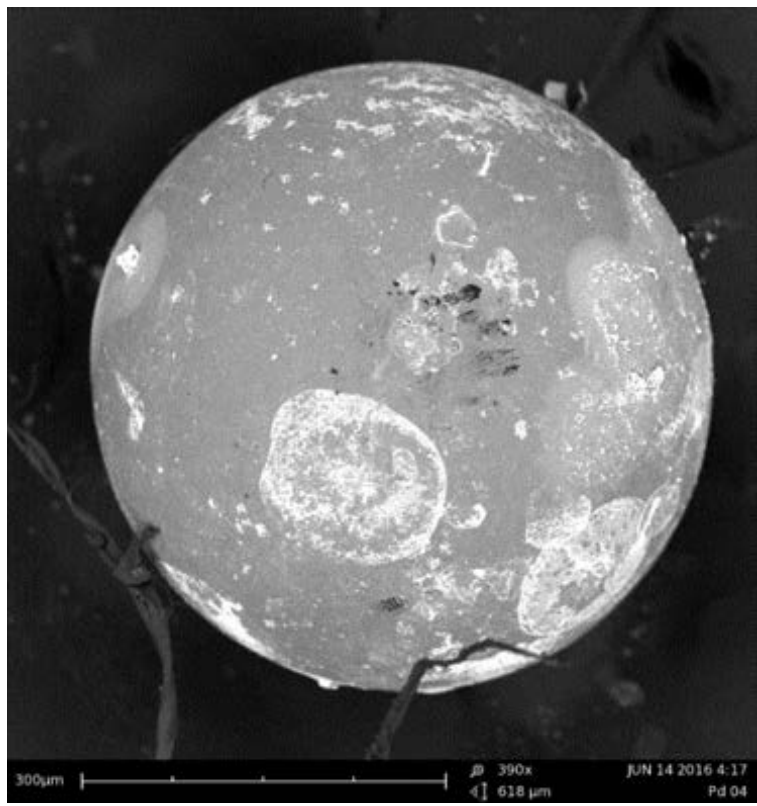

Figure 7. Palladium experiment at 24 hours.

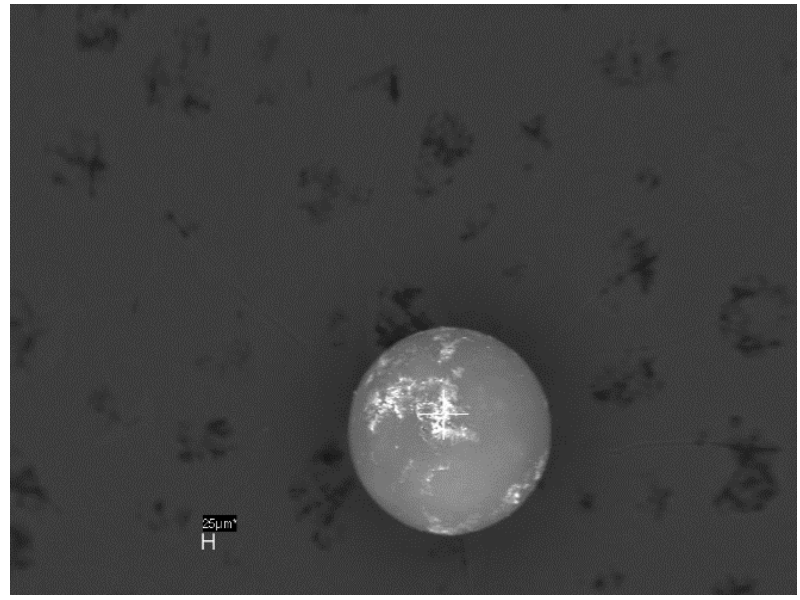

Figure 8. Palladium experiment at 48 hours. 


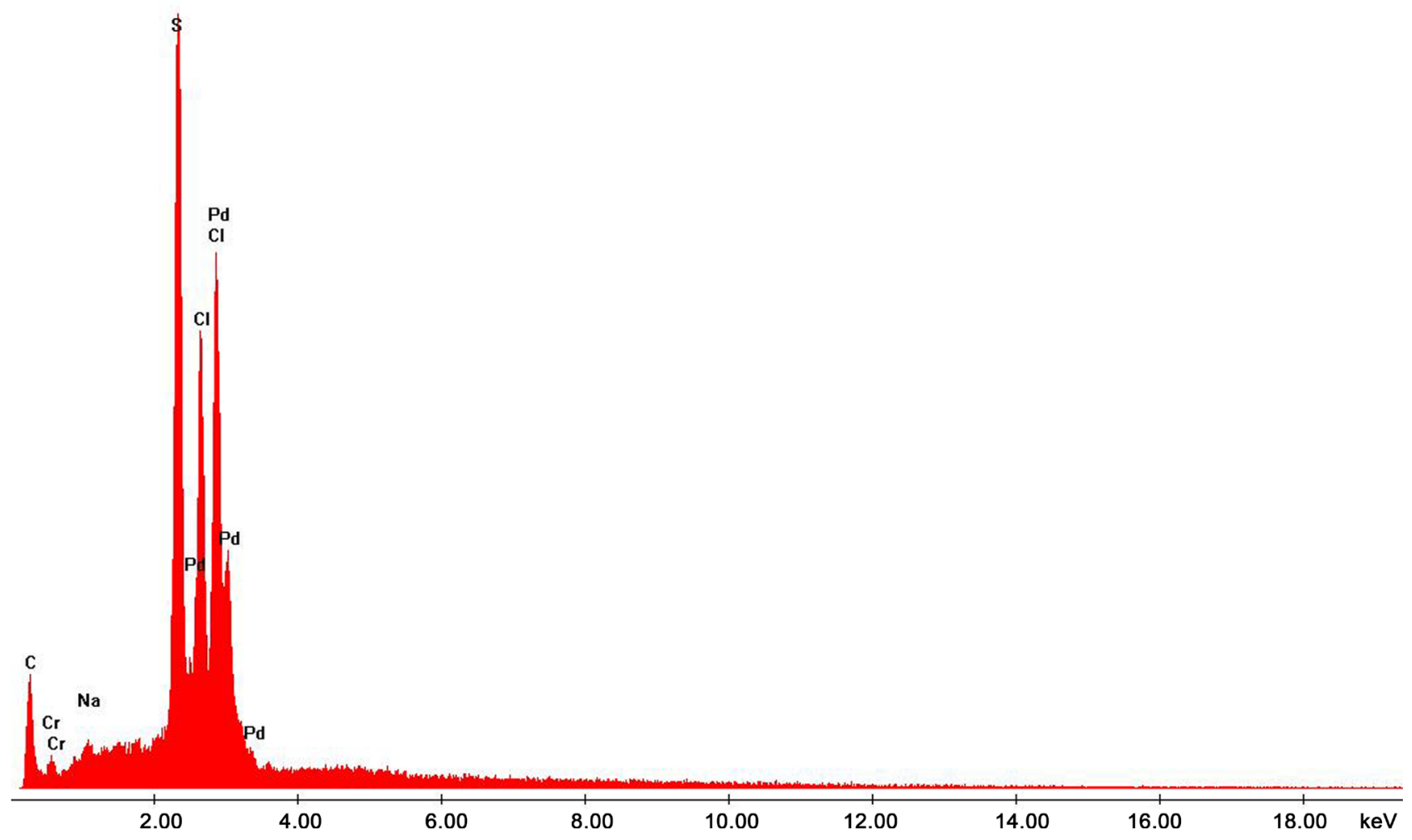

Figure 9. EDS of palladium on polystyrene resin.

Similar depositional structures are seen with other transitional and precious metals, where the final result is complete covering of the surfaces by a thick, platelike form. As Figure 9 shows, the deposits are chlorides, resulting from conventional redox reactions in the solution that cause surface plating of the reconstituted metal chloride form.

\subsection{Characterization of Phenomena through Molecular Modeling}

MarvinSketch, Version 7.0 (ChemAxon) was used for molecular modeling. The model's algorithms for conformational optimization and stress localization are proprietary and are discussed in the company's literature. In this section we expand on the steps taken to model the stress state of the composite structure with metallic loading in a simplified state.

Molecular modeling uses algorithms and experimental data from multiple sources to predict how species will interact given structural and chemical constraints input by the user. The modeling in this project assumes the basic principles set forth by the MarvinSketch program and the governing proprietary algorithms. These principles include the best available information and incorporation of established theory regarding:

- Bonding type and conditions;

- Charge transfer; 
- Electron localization and densification;

- Conformational accommodations;

- Calculation of stress states.

There are limited publications on composite anomalies similar to those seen in this research program, making molecular modeling an important tool in attempting to ascertain the mechanisms of loading, the resulting structures, and the rupturing mechanisms.

Loading was occurring as nodules, island growths, sheets, upward tree and branch growth, and circular formations. Our simplified modeling assumed a base structure of polystyrene with no functional ligands. Since previous testing showed ligand structure appearing to play a major role in initial attachment but a minor role in subsequent unrestrained metallic surface growth, the primary consideration for this phase was the interaction of REE loading and base polystyrene structure. That structure is a limited polymer unit consisting of a finite amount of monomer units.

Modeling of the REE loading proceeded as:

1) Point attachment;

2) REE bridging;

3) REE growth and metallic formation;

4) Halting REE loading after sufficient (as deemed by the user) metallic growth and outward repetition-simulating the loss of available material.

Modeling was in 3-D view and the structure optimized using the proprietary applications of MarvinSketch.

It was initially found that the structure is able to accommodate increased metallic formation of the REE. The resulting setup appeared to act like a metallic back-donation bonding structure capable of transferring electrons around a bulk structure for stabilization. This is consistent with the transition from the highest occupied molecular orbital to the lowest unoccupied molecular orbital (HOMOLUMO) and subsequent metallic formations after electronic failure. Modeling was expanded to basic silica and polystyrene structures to show the stages of progression and the complexity of the molecular modeling. The f-orbital systems serve to increasingly complicate matters due to their sizes and multiple coordination sets.

Although our modeling included both polystyrene- and silica-based polymer structures, polystyrene is the focus of this report. The polystyrene structure was modeled with loading of dysprosium, a generalized polystyrene that is nonspecific to the composites used in this research program. Steric conditions were optimized using the program's algorithms to seek a low-energy state. Subsequent calculations were on the basis of this low-energy state.

The loaded polystyrene structure has relatively uniform outer species distribution of charge and electronegativity, except for oxygen acting as a concentrator. Xiong et al. (2012) attribute this to lanthanide ions having "a lack of stereo chemical preference, a handful of high coordination numbers and small energy 
variations among their various coordination geometries." [4]. The parallel nature of the charge and electronegative concentration establishes interior vacancies as the polystyrene conforms to accommodate the incoming REE species. The network is preferentially attaching more REE atoms, rather than stopping adsorption at point attachment, modeling the HOMO-LUMO transition. The electronic balance is coming from the internal network, including vacancies. Because conformational changes result in steric stress, a fracture plane is established. The fracture plane's epicenter is on the oxygen species that has been placed in an increased stress state. The first breakage event will result in a cascade of failures along the polystyrene backbone as the electronic transfer network is disrupted. The exact break point is the result of a number of factors, including steric considerations, volumetric and 3-D conformation, and internal force energy. This breakage appears to cause crazing, in which an REE-loaded outer shell breaks away from the inner particle.

The polystyrene system, depicted in Figure 10, occupies a van der Waals volume of $3535.33 \AA^{3}$. Energetically, the setup has a Dreiding energy of 1763.74 $\mathrm{kcal} / \mathrm{mol}$ (basic cyclohexane is $7.72 \mathrm{kcal} / \mathrm{mol}$ [as calculated by MarvinSketch]). The energy state indicates that the limited loading on the short-range polystyrene backbone is leading to heightened stress. Increased loading and geometric

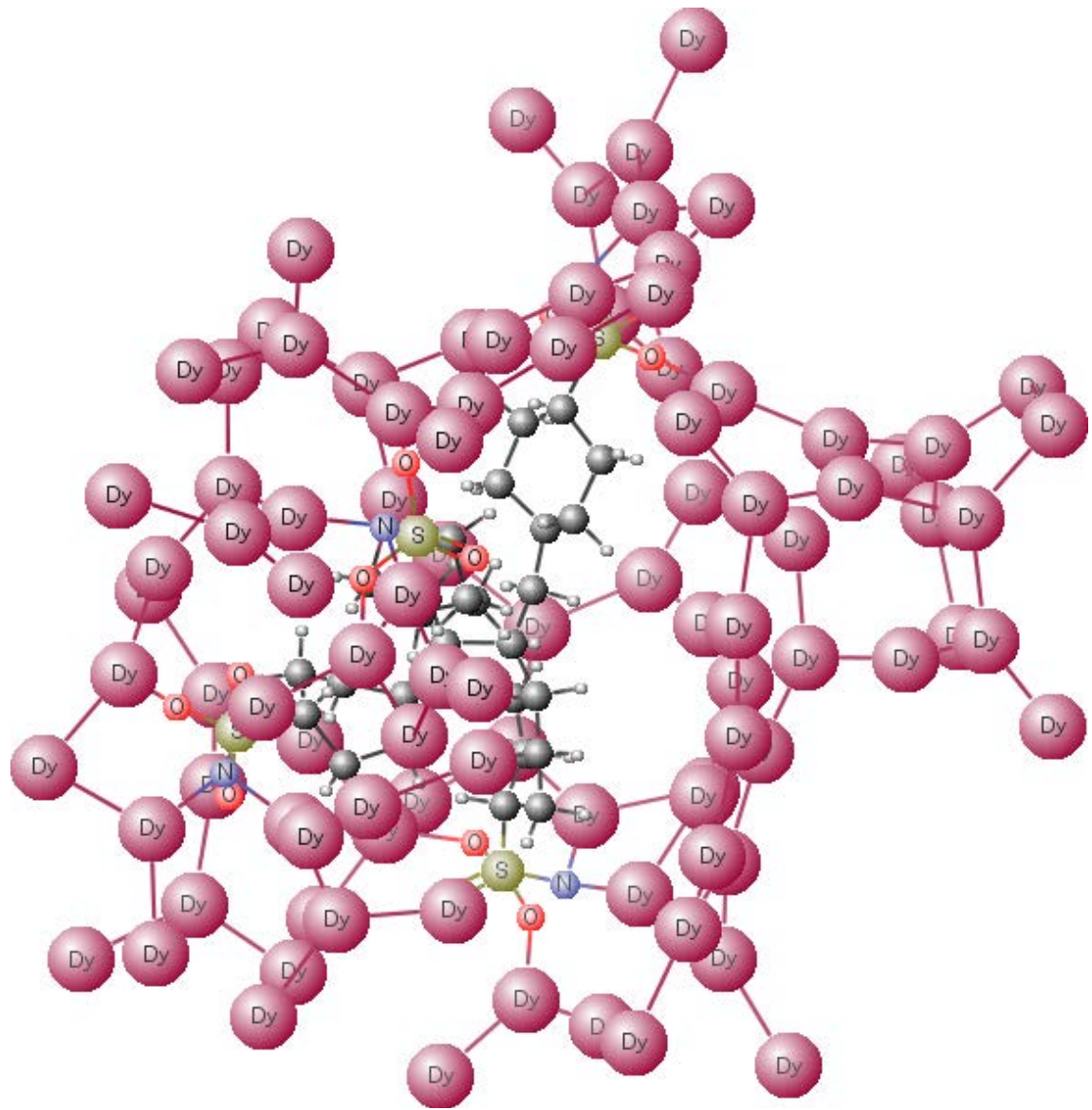

Figure 10. 3-D molecular model of polystyrene loaded with dysprosium. 
considerations in pore space conformation serve to multiply stress effects.

\subsection{Mechanisms}

Two important composite modifications are evident from SEM analysis: particle rupture and metallic deposition onto the particle. SEM images show a progression of REE loading on the ion exchange/chelating resins, subsequent metallic formation, and in many cases, particle fracture.

Typical loading mechanisms, for most adsorption purposes, are point attachments at specific engineered sites. Continued interaction is not known to widely occur. Functional site REE attachment on the ion exchange/chelating resins phase from point loading to self-assembling metallic formation, in some cases unrestrained.

These formations indicate a second adsorption mechanism. The presence of metallic formations, is indicating that both organometallic adsorption and metallic adsorption are occurring. Instances of this type of metallic adsorption and subsequent growth have not been previously reported. The phenomenon is thought to be the result of free electron coordination with REE species, setting up an electron pump created by interactions between the highest occupied molecular orbital and the lowest unoccupied molecular orbital (HOMO-LUMO) that changes band gaps and conduction behaviors.

After sufficient surface deposition of REE, a secondary metallic mechanism of delocalized charge is activated, with unrestrained surface growth of metallic species. The codependence of these mechanisms is centered on the ability to transfer an electric charge. The polymer structure initially acts as both a source of electrons and a dispersion network routing electrons in need of stabilization to areas at the surface. Localized stress states and charge centers result due to the random nature of the underlying polymer network.

Modeling has shown that stress states or charge localization occurs in the interior of the polymer chains. This process is analogous to oxidation of polymeric systems. HOMO-LUMO excitation and subsequent conduction in an excited polymer network leads to localized stress states, which causes polymer corrosion. As the metallic loading begins, stress localization occurs, followed by polymeric crazing, and finally breakage along preferred attachment formations or substrate weaknesses.

Oxidation-reduction is occurring due to reduced metallic formation, but there are no apparent oxidized species in the solution or polymer. A five-step process is proposed to explain the presence of metallic species:

1) The HOMO-LUMO interaction in polymers acts as an initial organic semiconductor process.

2) Energy associated with the REE-organic bond drives the electronic potential available from the nonreacted end-mers on the polymer chains.

3) The electronic interacts with REE orbitals once sufficient metal atoms are on the polymer surface as coordinated species. 
4) Reduction occurs directly on the surface rather than in the near-surface solution as the electrons are pumped in manner similar to fuel cell electron transfer.

5) Deposition rates increase as bonding sites multiply.

The dynamic nature of metallic interaction with organic frameworks is addressed to some extent in the literature. Gold nanoparticles were controlled through strict procedures using capping agents and reducing agents, and block copolymers displayed a metal-reducing function and self-stabilizing ability [8]. The reduction of gold can be analogous to the reduction of REE. Self-reducing metal species and the ability to self-regulate chemical stability is a basis for arguments. The variable coordination numbers exhibited by REE metals give them a higher affinity for hard donor atoms, increasing the likelihood for stability [9]

The f-orbital's ability to accommodate multiple conformations is a primary driver for creating the electronic network that stabilizes REE on polymeric structures in the metallic state.

The deposition of metals versus chlorides in these cases can be seen clearly. Chlorides are also seen in a small percentage of the SEM EDS analyses. In some cases, both types of depositions occur. It is thought that deposition may transition to a solution-reduced chloride when the source of electrons availed in the polymeric ion exchange resin structure is consumed or when the internal network enabling electron transfer within the ion exchange resin is disrupted. Oxygen did not appear to play a part in metallic deposition.

\section{Conclusions}

Attachment and growth occurs on the atomic scale then transitions to a nanoscale phenomenon but can extend to the microscale or macroscale. Extension is limited only by the capability of the internal networks, both ionic and electronic, to transfer species within the structure, and the ability to supply REE to the surface.

HOMO-LUMO theory and the f-orbitals in organometallic frameworks may explain the type of electron transfer and on-surface reduction seen in this project. A key component to the problem presented is that the bonding and electron transfer between REE and organic frameworks is centered on the f-orbital. The f-orbital is little studied and its interaction on the HOMO-LUMO front is little understood.

This work does not propose a new framework for electronic sharing or bonding, but rather a possible explanation for the occurrences being seen.

Proof of concept was achieved, showing that REE can be recovered from pure solutions. Expansion of research must confirm recovery from leach liquor solution. Research diverged when observations were made of the composite materials behaving differently than behavior reported in interaction with transition elements. Cracking and agglomeration led to further experimentation and subsequent theories as to further research and implications. 
Further experimentation and analysis focuses on the ability to control the rate at which adsorption occurs. If rate can be controlled, then the adsorption could preferentially be turned off and on. This switch will allow self-assembly of multiple species on a single particle through advanced processing. Applications such as an in-situ alloy formation process can then be developed.

\section{Funding}

This work was supported in part by the U.S. Office of Naval Research [Project No. N000141210592] and the US Army Research Laboratory [Project No. W911NF-10-2-0025].

Research was sponsored by the Army Research Laboratory and was accomplished under Cooperative Agreement Number W911NF-15-2-0020. The views and conclusions contained in this document are those of the authors and should not be interpreted as representing the official policies, either expressed or implied, of the Army Research Laboratory or the US Government. The US Government is authorized to reproduce and distribute reprints for Government purposes notwithstanding any copyright notation herein.

\section{References}

[1] Grasso, V.B. (2012) Rare Earth Elements in National Defense: Background, Oversight Issues, and Options for Congress; Congressional Research Service Report for Congress.

[2] Figgis, B.N. and Hitchman, M.A. (2000) Ligand Field Theory and Its Applications. Wiley-VCH.

[3] Qui, J., Wang, Z., Li, H., Xu, L., Peng, J., Zhai, M., Yang, C., Li, J. and Wei, G. (2009) Adsorption of Cr(VI) Using Silica Based Adsorbent Prepared by RadiationInduced Grafting. Journal of Hazardous Materials, 166, 270-276. https://doi.org/10.1016/j.jhazmat.2008.11.053

[4] Wang, Z., Ye, C., Wang, X. and Li, J. (2013) Adsorption and Desorption Characteristics of Imidazole-Modified Silica for Chromium(VI). Applied Surface Science, 287, 232-241. https://doi.org/10.1016/j.apsusc.2013.09.133

[5] Xiong, C., Zhu, J., Chen, S. and Chen, Q. (2012) Adsorption and Desorption of Praseodymium(III) from Aqueous Solution Using D72 Resin. Separation Science and Engineering, 20, 823-830.

[6] Zheng, Z. and Xiong, C. (2011) Adsorption Behavior of Ytterbium(III) on Gel-Type Weak Acid Resin. Journal of Rare Earths, 29, 407-412. https://doi.org/10.1016/S1002-0721(10)60469-3

[7] Yao, C. (2010) Adsorption and Desorption Properties of D151 Resin for Ce(III). Journal of Rare Earths, 28, 183-188. https://doi.org/10.1016/S1002-0721(10)60324-9

[8] Sakai, T., Horiuchi, Y., Alexandridis, P., Okada, T. and Mishima, S. (2013) Block Co-Polymer Mediated Synthesis of Gold Nanoparticles in Aqueous Solutions: Segment Effect on Gold ion Reduction, Stabilization, and Particle Morphology. Journal of Colloid and Interface Science, 394, 124-131. https://doi.org/10.1016/j.jcis.2012.12.003

[9] Li, B., Wen, H.M., Cui, Y., Qian, G. and Chen, B. (2015) Multifunctional Lanthanide Coordination Polymers. Progress in Polymer Science, 48, 40-84. https://doi.org/10.1016/j.progpolymsci.2015.04.008 